a bloodless surgical field and to assess the chordee by artificial erection. ${ }^{[1]}$ Despite a number of popular tourniquets used in penile surgery, there are no clear guidelines for the safe use of tourniquets. Different types of material are used for a penile tourniquet such as a red rubber catheter, soft latex catheter, a Penrose drain clipped around the base of the penis using artery forceps, rubber band, and the rolled rubber glove, or twisting a silicon vascular band around the base of degloved penis by using a clip [Figure 1].

To minimise the risk of excessive tourniquet pressure, current recommendations advise the use of the lowest pressure necessary to maintain haemostasis. According to data the Penrose drain and rolled glove with clamp methods exerted high pressures in excess of $300 \mathrm{mmHg} \cdot{ }^{[2-4]}$ Thus the clamp (artery forceps) to tighten the tourniquet should be avoided.

\title{
DESIGN OF THE TOURNIQUET
}

One $\mathrm{mm}$ thick flat, soft silicone sheet is taken. Depending upon the size of the penis, $5-10 \mathrm{~mm}$ wide strips are cut from the silicone sheet with $1 \mathrm{~mm}$ increments and $10-15 \mathrm{~cm}$ in length.

A total of 2 tubectomy rings are threaded over the silicone strip with the help of straight artery forceps to make the tourniquet [Figure 2]. The haemorrhoids rubber rings cannot be used because of its being oversized. The threading of the tubectomy rings is facilitated over the strips after lubricating with sterile jelly or distilled water. The first ring exerts the needed pressure to check bleeding, and the second one retains the position of the first ring and does not allow it to slip out.

Our penile tourniquets can be sterilised by any conventional method without any damage.

\section{APPLICATION OF THE TOURNIQUET}

\section{A simple and safe penile tourniquet}

Sir,

Tourniquets have been widely used in penile surgery, especially for hypospadias surgery, for the last five decades. The purpose of these tourniquets is to obtain
The tourniquet is passed through the tip of the penis to the base of the penis. The assistant squeezes out the penis after applying the gauge sponge, and tubectomy rings are advanced over the silicone strip toward the base of the penis to fasten the tourniquet. Now the surgeon makes a small cut at the proposed site of the incision. If bleeding is present, the ring is further advanced over silicone strip just to stop bleeding. If there is no bleeding, the ring is just

Indian Journal of Plastic Surgery January-April 2015 Vol 48 Issue 1 
withdrawn a little bit to observe the bleeding point and from here it is slid toward the base of the penis to stop it. The second ring (retaining ring) is advanced to hold the first ring in position to avoid its accidental slippage after getting lubricated with blood during the operation.

Now the tourniquet is on with appropriately safe pressure [Figure 3]. At this point, the tourniquet starting time is noted down. If the operative time exceeds $45 \mathrm{~min}$, the tourniquet is released for $5 \mathrm{~min}$, and then reapplied as mentioned above.

The authors have operated 37 patients of hypospadias aging from 1.5 to 23 years in the last 4 years from April 2009 to October 2013 using this tourniquet. The authors applied these tourniquets up to $45 \mathrm{~min}$ at a stretch without any untoward side effects in the patients such as skin, glanular necrosis, and major wound dehiscence. The tourniquet is dynamic, and it just checks the bleeding without over-tightening the organ. The urinary catheter can

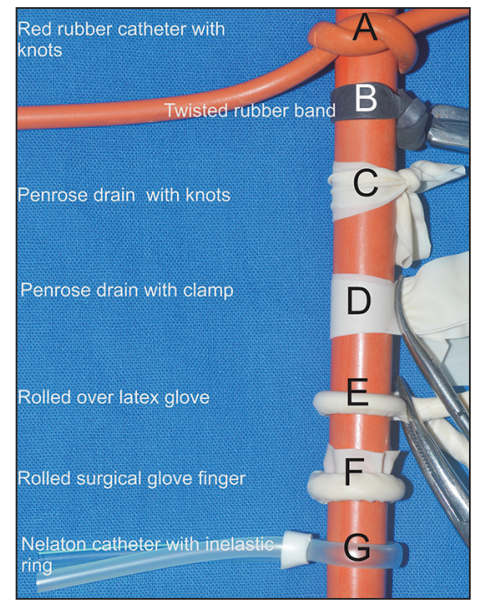

Figure 1: Different type of penile tourniquets are being used: Red rubber catheter with knots, twisted rubber band, Penrose drain with or without knots, rolled over the glove and glove finger, Nelaton catheter

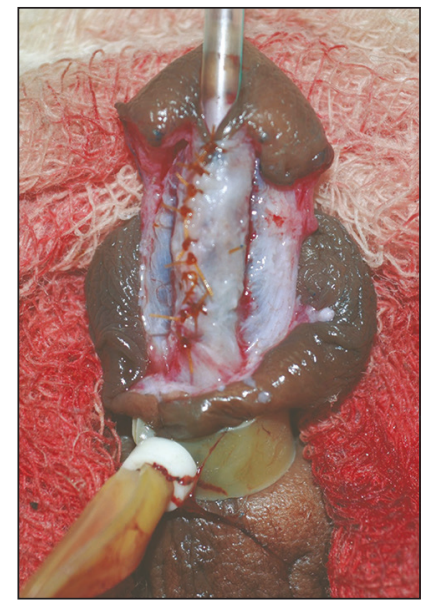

Figure 3: Authors' design of the penile tourniquet in use be easily passed through the urethra without unfastening it. In severe proximal type of hypospadias, this tourniquet is applied on the degloved penis at its base to obtain haemostasis while working on vascular glans.

Controlled pressure can be applied easily and safely with this tourniquet to check the bleeding effectively during penile surgery. The rigid tubular structure or any other rigid strip along with haemostat, used in existing tourniquets, occupy the operative area and clutter the operative field. The long tail of this tourniquet strips reminds the operator to remove it at the end of the surgery. The strip is soft and does not hinder in the movements of the instruments. The tubectomy rings are also soft and are just sitting over the silicone strip to avoid sharp pressure on the penis.

The pressure, in case of flat strip tourniquet, equally distributed in the whole of the contact area on the penis and thus local tissue damage is lesser as

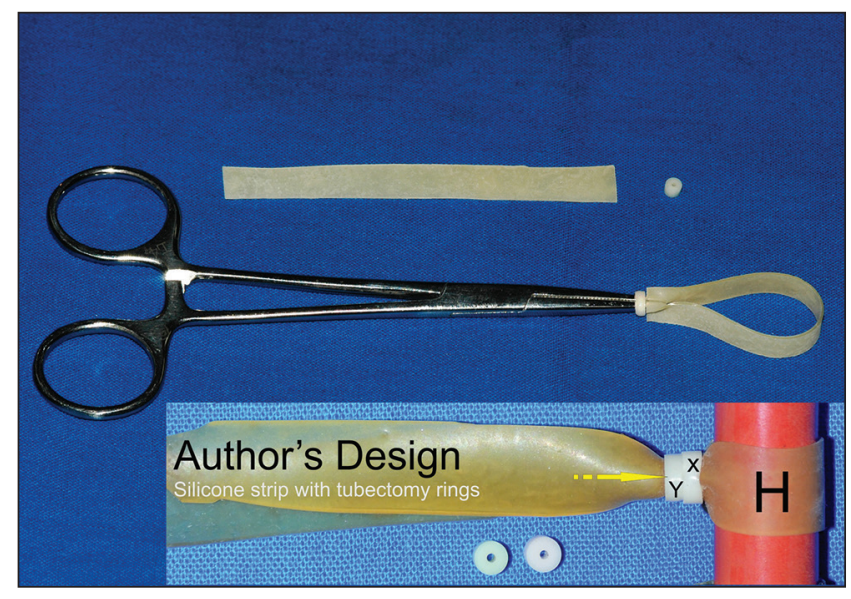

Figure 2: Authors' design of the penile tourniquet

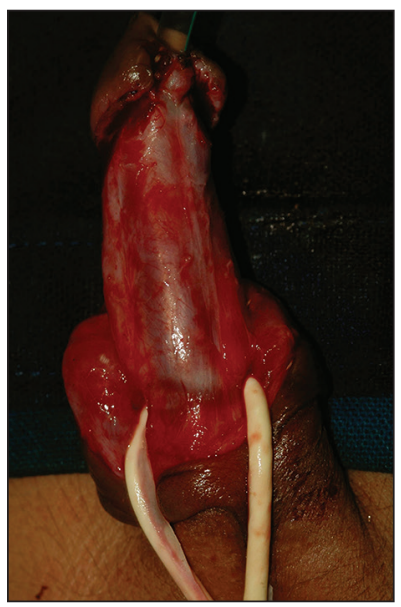

Figure 4: Local tissue damage using tubular tourniquet (rolled glove tourniquet with more than recommended application time and pressure) 
compared to tubular tourniquet [Figure 4]. The pressure exerted by tubular tourniquet is not uniform in the contact area but, it is maximum in its centre. Hence, our tourniquet design may be considered safe. However, the ill effect of the pressure can be minimised by using optimum force to generate adequate pressure.

This tourniquet is simple and safe. It provides a clear surgical field of enhanced visibility since it is made of flat, silicone strip of appropriate width that reduces sharp pressure on soft tissue, vessels and nerves.

\section{Devendra Kumar Gupta, Shruti Devendra}

Department of Plastic Surgery, Devendra Hospital and Yuva Cosmetic Clinic, Bareilly, Uttar Pradesh, India

Address for correspondence: Dr. Devendra Kumar Gupta, Devendra Hospital and Yuva Cosmetic Clinic, 55 Prabhat Nagar, Bareilly - 243 122, Uttar Pradesh, India. E-mail: dr.dkgupt@gmail.com

\section{REFERENCES}

1. Ossandón F, Ransley PG. Lasso tourniquet for artificial erection in hypospadias. Urology 1982;19:656-7.

2. Parsons BA, Kalejaiye O, Mohammed M, Persad RA. The penile tourniquet. Asian J Androl 2013;15:364-7.

3. Hixson FP, Shafiroff BB, Werner FW, Palmer AK. Digital tourniquets: A pressure study with clinical relevance. J Hand Surg Am 1986;11:865-8.

4. Lahham S, Tu K, Ni M, Tran V, Lotfipour S, Anderson CL, et al. Comparison of pressures applied by digital tourniquets in the emergency department. West J Emerg Med 2011;12: 242-9.

\begin{tabular}{|l|l|}
\multicolumn{3}{|c|}{ Access this article online } \\
\hline Quick Response Code: & Website: \\
\hline
\end{tabular}

